east, and west of the inflammatory swelling-leaving a forceps attached to each. The "south" piece is the longest and extends well into the pelvis ; if the appendix is closely adherent to the outer part of the iliac fossa, there may be no room for more than a short "west" piece. While introducing the gauze I always keep my left index finger placed lightly upon the swelling to prevent traction being exerted upon it while the gauze is pushed into place with long dressing forceps. Unless this precaution is observed, it is easy for the gauze to carry along with it a portion of intestine constituting the wall of the inflammatory mass, and so to break down liniting adhesions and flood the area with pus before packing is completed. Having, then, securely isolated the inflamed area from the rest of the peritoneal cavity, I open up the mass with my finger, wiping away any pus as fast as it escapes until the abscess cavity is dry, $o_{i}$, if no pus be present, searching for the appendix itself. The factors which influence me in deciding when to search for the appendix in the presence of a localized abscess have already been referred to. Wherever possible I prefer to treat the appendix as I do in "interval" operations-that is, crush and ligate its base and bury the stump in the caecal wall with a purse-string suture. In many cases, however, where the base of the appendix is situated deeply, it is not advisable to draw up the caecum sufficiently to pass a purse-string suture, and I have no hesitation in leaving the stump simply ligated, though here I invariably employ drainage. The gauze packs are next removed in the inverse order of their insertion, the "east" piece usually bringing with it a little of the omentum, which I tuck snugly around the drainage tube when such is used. Flushing with saline I now never employ except for cleansing large abscesses which have been reached without exposure of the general peritoneal cavity. I have already referred to the treatment of desperate cases of diffuse peritonitis and the importance of not attempting too much.

\section{THE INGUINAL OPERATION FOR THE RADICAL CURE OF FEMORAL HERNIA.}

By CHARLES A. MORTON, F.R.C.S.,

PROFESSOR OF SURGERY IN THE UNIVERSITY OF BRISTOL; SENIOR SURGEON TO THE GFNERAL HOSPITAL AND THE CHILDREN'S
HÚSPITAL, BRISTOL.

THE inguinal method of operation for the cure of femoral hernia seems now to be very frequently employed. My attention was first called to it by the paper of Mr. T. E. Gordon in the British Medical Journal, 1900, vol. i, p. -1338 , at a time when I was myself planning some method for closing the crural canal at its upper end, and $I$ began to perform the operation of radical cure of femoral hernia by this method in January, 1903. Since then I have operated on 27 cases in this .way up to the end of June, 1911, and I have endeavoured to trace the first 12 of these cases. They were all operated on more than six years ago, and therefore there has been plenty of time for late relapse. It is stated that when relapse occurs after operation for femoral hernia it is usually late.

The statistics given to show the results of operation for radical cure of hernia are not usually satisfactory. We are generally told that so many cases have been operated on by some special method and only. so many relapses are known ; or if the cases are examined and found free from relapse, the time after operation at which such an examination is made is : not given for all cases. What we really want to know is not whether few cases returned to the surgeon with relapse, for, of course, relapsed cases might quite likely not return to the surgeon who had operated, or whether so many cases were found free from relapse when examined shortly after the operation, but what proportion of patients have been found free from relapse some years after the operation. Even if they are free from relapse many years after the operation, that does not, of course, prove that they will never get a relapse, but we must have some time limit. But every one who has tried to obtain the result of treatment years after an operation knows how extremely difficult it often is to trace such cases amongst hospital patients.
They move so frequently, and where they move to is not generally known in the neighbourhood where they previously lived. I have been more fortunate in tracing my first twelve patients operated on by this method of radical cure than I expected. Two out of the twelve died; one of them was an elderly man who died a week after the operation (which was for strangulated hernia followed by "radical cure") from bronchitis, and the other died nine months after the operation, of heart disease and without any recurrence of the rupture. In three cases my letters were returned through the "dead letter" office and seven replied. All these seven stated that they were free from recurrence, though one male had developed an inguinal hernia on the same side. I was able to examine four myself. Thus all the cases (seven) it was possible to trace, out of the twelve, were free from recurrence six years after the operation. In looking through the records of these cases, I have been struck with the small proportion of radical cures of femoral hernia which I have done, compared with inguinal hernia. $I$ find $I$ have only done radical cure for femoral hernia in 47 cases, and in no less than 27 of these the radical cure was done after herniotomy had been done for strangulation, leaving only 20 cases of operation for the radical cure of femoral hernia apart from strangulation. Some of the seven cases found free from recurrence after six years were radical cures done at the end of a herniotomy. I have not looked through my records to see in how many cases of inguinal hernia, which I have operated on for strangulation, I have done a radical cure, but I find that, whereas I have only operated in 20 cases of non-strangulated femoral hernia for radical cure, I have done so in 156 cases of non-strangulated inguinal hernia.

Although when considering the best method of closing the crural canal I came across the paper by Mr. Gordon, yet a little more than a year later, and before I began to practise the operation, Mr. Parry of Glasgow advocated it in a paper published in the British Medical Jouranal, 1901, vol. ii, p. 1136. I do not remember reading the paper at that time, but Mr. Parry had practised the method for four years before he published his paper-that is, he performed it for some time before Mr. Gordon did so. But unknown to both these surgeons, Lotheissen ${ }^{1}$ described a similar operation several years before, and both he and Parry were led to adopt this method for the radical cure of femoral hernia after they had employed it in the operation for inguinal hernia. : Parry brought the cord out through the crural canal and fixed the internal oblique and transversalis to Cooper's ligament, so as to form a barrier against descent of both forms of hernia. Lotheissen also fixed the conjoint tendon to Cooper's ligament in operating for combined inguinal and femoral hernia, and was thus led to adopt the method for femoral hernia alone.

The operation which I perform is not quite the same as the method described by Lotheissen, Gordon, or Parry, and $I$ think it will be well first to describe it and then to point out how it differs from them. I make an incision parallel to and very slightly above Poupart's ligament; in the position of the neck of the sac, and then carry it downwards at the inner end at almost a right angle to the part parallel with Poupart's ligament, but where the horizontal joins the vertical portion it is curved, not angular. Each limb of the incision is about 2 in. long. The flap of skin which is thus cut is then dissected up in the outward direction, exposing the sac, Poupart's ligament, and the lowest pait of the aponeurosis of the external oblique above it. The sac is now opened and the contents reduced. . It is better not to tie the neck in the thigh, as it can be tied higher up from above Poupart's ligament, when later on it is reached through the incision through the external oblique; but if the sac is large it may not be easy to pull it up through the crural canal, and thus it may be necessary to tie the neck of the sac in the thigh, and after removal of the rest of the sac simply return the tied neck through the crural canal. In any case, whether tied in the thigh or above Poupart's ligament, the sac is always removed: If it is removed from above Poupart's ligament, it should, I think, be separated from its surrounding fat and fascia by dissection in the thigh before it is drawn up through the crural ring. Hence the incision should be carried over the sac in the thigh by means of $a$ vertical limb, in the way. I have described. Having; then; separated the sac ready to be drawn up 
through the crural canal, or removed it after ligature of its neck, an incision is made through the external oblique just above and parallel with Poupart's ligament, as in Bassini's method of performing radical cure of inguinal hernia. It is not, however, necessary to carry this incision into the external ring. The transversalis fascia must be divided, and then either the ligated neck of the sac will appear if it has been returned from the thigh, or if it has not the neck of the sac will be identified and the whole sac, already separated from its surroundings in the thigh, must be drawn up through the crural canal, the neck tied so as to take into the ligature as much peritoneum as possible in order to obliterate the neck, and then the rest of the sac cut off. In the female the round ligament can be easily separated and drawn aside, and the cord can also be easily displaced without any harmful traction so as to get good access to the parts beneath, as I have found in several operations performed by this method in males. I may say that in thin patients of either sex there is no real difficulty about the operation if you know the anatomy of the part you are dealing with, but these structures do lie at a very great depth in very fat patients, and it is not easy to then get a good view of the deep part of the wound, and the difficulty in getting your needle to take the required curve at a very considerable depth and in a very narrow space may be considerable. Having dealt with the sac you must next define the crural ring, and particularly the ilio-pectineal line, with the origin of the pectineus muscle on it, covered by the thick pectineus fascia (the pubic portion of the fascia lata). This line of fascia is sometimes called Cooper's ligament. It should be split open parallel to the ilio-pectineal line, to the extent of the diameter of the crural ring, so as to expose the fibres of the pectineus muscle in order to obtain a raw surface to which the lower edge of the abdominal muscles can be united. It is, of course, necessary at this stage to carefully define the position of the femoral vein. I have never had any trouble from the deep epigastric vessels, but it is well to remember their position. I may have had to tie the deep epigastric vein when I have had to deal with venous bleed ing, but I have certainly never cut the artery. I find a metal spatula is the best form of retractor in the deep space. It holds aside the loose fat in a way the ordinary hook retractor does not do. As soon as you have made your incision in the edge of the pectineal fascia it is best to pass three silk stitches through the edge of the split fascia, and then to rethread the deep end of each stitch and carry it through the lower edge of the internal oblique and transversalis muscles, and by tying the knot fix the muscles to the cut edge of the fascia. It is easier to pass all these stitches first through the fascia and then afterwards through the muscles, than to include fascia and muscle in each stitch as it is passed. I generally draw down and fix to the fascia the muscle edge before it becomes the conjoint tendon. The latter never seems to me a firm enough structure to be used with advantage in this way. By tixing the abdominal muscles to the pectineal fascia a carrier is formed to the entrance of a hernia into the crural canal. It has seemed to me that $a$ weak spot was left after the operation just ozer the femoral vein, and that an incipient hernial sac might protrude over the vein round the outer edge of the new barrier, and thus enter the crural canal. In many of my operations I have therefore sewn the internal oblique to Poupart's ligament over the vein-that is I have carried the barrier further out-and I think in some cases this is a wise thing to do; but it does not seem necessary in all, as I sometimes find, after suturing the internal oblique and transversalis to the pectineal fascia the edge of the muscle is so closely applied to the vein as to make it seern impossible for a hernial sac to descend there. Gordon, in the account of his operation, advises the stitching of the internal oblique to Poupart's ligament above the crural ring. He says this is done "so as to pack down as much muscle fibre as possible against the opening, and also to relieve any tension that might be thrown on the primary stitches." This is no doubt a good plan, but what I suggest is making a union to Poupart's ligament further out, over the femoral vein, in some cases, Parry also sutures the conjoint tendon to Poupart's ligament as well as to the pectineal fascia.

I have not adopted the method of performing the radical cure of femoral hernia by stitching down Poupart's ligament to the pectineal fascia for two reasons: I have always been afraid of wounding the femoral vein with my outermost stitch, and I thought that if Poupart's ligament were fixed down to the pectineal fascia so as to occlude the ring in this way it might press on the femoral vein. But when you have exposed the femoral vein-or, perhaps more strictly speaking, the iliac vein-from above Poupart's ligament, as you do in this inguinal method of operating, and can see exactly where it lies, there seems no danger of puncturing it; and, moreover, you can satisfy yourself it is not injuriously pressed on when you fix Poupart's ligament to the pectineal fascia, so that in my last two opera. tions-not in any of the first 25-I have stitched Poupart's ligament to the pectineal fascia, and have thus made the operation a combination of the method of Lotheissen and that of Lockwood and others. I have not done so because Lotheissen's operation has been a failure, but because with the ring expcse 1 from above it has bean so simple and safe to pass these extra stitches, and it seems to me that every extra barrier to further descent of the hermia must be an advantage. In many of my earlier cases I drew together with a purse-string suture the fascia around the saphenous opening, but this, of course, is of no great value. The inguinal operation is completed by sewing up the opening in the external oblique as in Bassini's operation.

Although for the reasons I have already stated I have not used any method of radical cure which consists in union of the wall of the crural canal by stitches passed from below, I have turned up the flap from the pectineus muscle advocated by Sir Watson Cheyne. But this always seems to me a closure of the canal at the wrong end, and I was planning closure of the upper end when I came across Mr. Gordon's paper. The closure of the crural ring rather than the saphenous opening is, of course, the great advantage of the inguinal operation.

I must now point out in what way the method by which I perform this operation differs from that of the three other surgeons who have described it. Lotheissen does not make any incision downwards over the sac, but only one parallel with Poupart's ligament, and the sac is generally dragged upwards into the space opened by the incision through the external oblique. But he says this may not be possible if the sac is large, and it cannot be possible if the hernia is irreducible. In such cases he simply draws down the lower edge of the inguinal skin incision, so as to expose the sac. It seems to me that better access is given to the sac by making a downward limb to the incision, and that a preliminary separation of the sac from its surrounding fascia in the thigh makes it easier to drag it up through the ring. I think it would be a great mistake to tie the neck of the sac first from the thigh and then retie it when the sac had been drawn up through the ring so as to get a ligature on it higher up, for some part of a coil of intestine might be lying in the neck above the first ligature, and so be included in the second. And if the first ligature were removed before another was put on, it would be difficult to get enough of the edge of the sac into the second ligature to make it hold well, for we should then have to deal with a fairly large opening in the peritoneum. But this might, of course, be sewn up, as one does with a very wide neck in operating on an inguinal hernia.

The method which I have adopted differs slightly from Parry's in the form of the incision, but more essentially in that I always remove the sac, as it seems to me there is nothing to be gained by drawing it up and leaving it under the abdominal wall. The tied neck can always be drawn up under the internal oblique and transversalis, out of the line of descent of the hervia, as some of us do in operating for inguinal hernia. Mr. Gordon's method and my own are almost identical. I have already explained that in some cases I made the union of the internal oblique to Poupart's ligament further out, that is, over the femoral vein, and that in the last two cases I have operated on I have sewn Poupart's ligament down to the pectineal fascia also.

At a meeting of the Section of Surgery at the Royal Society of Medicine, ${ }^{2}$ Mr. C. H. Fagge recorded his experiences of this inguinal operation in more than 30 cases. $\mathrm{He}$ was able to examine 19 out of 29 a short time after recovery from the operation, and found only 3 recurrences. All were, however, operated on as late as 1910, so that 
sufficient time had hardly elapsed to judge of the results. Mr. Fagge speaks of the advantage of this method of operation in strangulated femoral hernia, and he suggests that Gimbernat's ligament may be divided outside the sac through the inguinal incision in the external oblique. In division of the constricting band causing strangulation outside the sac, either in inguinal or femoral hernia it is necessary to be careful that while one's attention is given to this division, a very small piece of strangulated intestine-perhaps only a Richter's hernia-does not slip back unobserved as soon as the constriction is divided, and therefore before we have time to malke the examination of the groove of constriction for perforation, which is so important to do.

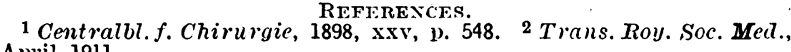
April, 1911.

\section{A CASE OF ADDER BITE.}

BBY

ERNEST F. CLOWES, M.R.C.S., L.R.C.P. WOTTON-UNDER-EDGE.

CAses of adder bite are of sufficient interest to warrant clescription, because of their rarity and the very alarming symptoms produced.

F. B., aged 28, a man enjoying excellent health and of good physique, was walking through some woods in search of gras snakes on June 16th, 1911 . He was quite unaware of the fact that the ordinary viper (Vipera berus) was to be found in every county of England, Scotland, and Wales. In a shody path he suddenly came across what he thought was a darli coloured grass snake; he picked it up, handled it, and noticed a V-shaped mark on its head, also that it was of a dirty chocolate-brown colour. After examining it for two or three minutes, he decided to take it home, and in order to get a box out of his pocket, he had to change the snake from his right hand to his left. Whilst doing this, the reptile suddenly bit him over the first phalanx of the left thumb. He dropped the snake, and says he felt darting pain shoot np the arm to his shoulder. After this he carnot pive a very clear account of his movements, but this he ber being found by some woodmen, who gave lim whisky anadvised him to suck the wound. He was removed to his home, advised him to suck the wound. He was removed to

I found him lying down, very pale and collapsed, and quite pulseless. His pupils were dilated, the left more than the pulseless. His pupils were dilated, the left more than the right; the sclerotics were pearly white and glistening. The
breathing was shallow and jerky; he was rather confused breathing was shallow and jerky; he was rather confused throat. The left thumb and hand was swollen, but the swelling was limited at the wrist.

I immediately applied a tight ligature abont 3 in. above the wrist, and soaked the whole hand in a strong solution of Condy's fluid. The thumb had two small punctured wounds about in apart on the dorsal aspect of the first phalanx; the wounds looked like small pin pricks. I made a deep crucial incision over the wounds, and allowed it to bleed freely, the wound was then filled up with crystals of potassium permanganate, and dressed with double cyanide gauze. A mixture of digitalis, compound spirit of ether, and ammonium carbonate was given, and a hypodermic of strychnine $\frac{1}{36}$ grain. Hot water bottles and a hypodermic of strychnine $\frac{1}{36}$ grain.
were applied to the feet, legs, and abdomen.

He rallied somewhat after this treatment. The pulse was 140 ; respirations 26 , shallow and jerky; temperature $96.6^{\circ} \mathrm{F}$. He complained of pains shooting like "electric shocks" down his spine, legs, and arms. His whole body was hyperaesthetic; so much so that he did not like the bed-clothes to touch him. He could only move his legs and arms feebly, the knee-jerks were absent in both legs, but slight plantar reflexes were present. He complained of difficulty in swallowing, and had a constant desire to pass water, although he could not do so. The corneal reflexes were decidedly sluggish. The pupils reacted to light and accommodations, but the left was more dilated than the right. He said that he could not see properly, everything
looked misty, and he could not tell the difference between a red and yellow rose when they were shown to him.

The swelling of the hand was now much increased, and there was great pain in the arm. I gave him a hot rectal saline injection and decided to infuse normal saline solution into the axillae. As I thought some of the pain in the arm was due axillae. As I thought some of the pain in the arm was due to the tightness of the ligature round the wrist, I loosened it
slightly, but almost immediately the patient cried out with slightly, but almost immediately the patient cried out with pain, which he said shot down his spine and legs. The pain was followed by vomiting and he was utterly collapsed. Another hot rectal saline and more of the ether mixture and a hyporallied.

As the patient's condition did not improve, local injections of sterile 5 per cent. permanganate solution at the edge of the oedematous area were made upon the suggestion of Professor Walker Hall; three separate injections were made at various sites round the limb; normal horse serum was also proposed to be given in $10 \mathrm{c.cm}$. doses three times daily. After the first dose of normal serum I waited for two hours, and then loosened the ligature again. There was some pain in the dorsal spine, and also slight pains down the legs, but no nausea or vomiting or collapse followed. At 8.40 p.m. the pulse was 110 . Respirations 20 , temperature $100.6^{\circ} \mathrm{F}$. The patient now passed some urine $(6 \mathrm{oz}$.); it was dark and smoky coloured; reaction acid; specific gravity 1025; albumen and blood present. Hot rectal salines were administered every three hours, and the ether mixture every two hours; nourishment in the shape of soups, milk, and egg-flip was given freely. An endeavour vas made to procure antivenene, but it could not be obtained in time for administration.

At 11.30 p.m. he was fairly comfortable, but complained of some pain in the forearm and shoulder. His pulse was 100, respirations 20 , temperature $101.8^{\circ} \mathrm{F}$. I loosened the ligature again, and no bad symptoms supervened. I left him for the night with instructions for the rectal salines and the ether mixture to be continued

I saw him early in the morning of June 17th. He had slept a little, but had suffered some pain in the left arm and shoulder. He had passed about $10 \mathrm{oz}$. of blood-stained urine during the night. The whole arm from the hand to the shoulder was now intensely oedematous, and the oedema extended slightly over the chest, neck, and back. The hand and arm were bluish red in colour, and the lymphatics stood and arm were bluish red in colour, and the lymphatics stood out as bright red lines. He complained of headache, and he could not ret distinguish colours. The left pupil had nearly regained its normal size. He still had much loss of power in the legs, and the knee-jerks were absent, but the plantar reflexes were stronger. Pulse 90; respirations 18 and normal in character ; temperature $100^{\circ} \mathrm{F}$. From then onwards the patient made an uninterrupted recovery. Knee-jerks appeared on June 20th. The urine was free from blood and albumen on June 21st, and all oedema of the arm had subsided by June 28th. For about a month the patient had a good deal of stiffness in the hand and fingers, but otherwise was quite well.

The case gains in interest from the fact that the patient was of more than average intelligence, and did not manifest any hysterical tendencies. Except during the period of collapse, he was able to give a succinct account of his symptoms. The transference of the venom appeared to be limited to the superficial lymphatics, and to occasion a considerable amount of reaction along their path. When the ligature was loosened, the resultant pain followed too rapidly to be due to any circulation of the poison; the suggestion lay near to hand to regard the condition as associated with a primary.inhibition of nervous impulso and the general systemic pains as referred in character. The normal horse serum appeared to play a part in reducing the effect of subsequent removal of the ligature and the admission of small doses of the poisoned oedema fluid distal to the ligature into the general system, for even after the first dose there was less pain when the ligature was slackened.

\section{A CASE OF \\ RUPTURED SPLEEN : SPLENECTOMY : RECOVERY.}

By HENRY JOY CLARKE, Jun., M.A., M.B. B.C.CANTAB., M.R.C.S.ENG., L.R.C.P.LOND.,

HONORARY SURGEON TO THE DONCASTER ROYAL INFIRMARY.

THE following case upon which I operated presents several points of interest. The patient, a farmer, aged 52, was admitted into Doncaster Royal Infirmary on Thursday, July 20th, 1911, at 1 p.m.

History.

On July 18th, at 8 a.m., he fell from a hayrick about $12 \mathrm{ft}$. high on to his left side across a stone wall. He continued at his work until 9 a.m., and then went home to bed suffering a good deal of pain. In the afternoon he felt better, and got up for a little. About 10.30 p.m. the pain became worse; it was situated in the pit of his stomach and round the left side. On the folin the pit of his stomach and round the left side. On the following morning he sent for Dr. Chambers, but by this time the pain had become easier. On July 20th, Dr. Chambers, thinking that the man was suffering from internal haemorrhase, and that his spleen might be ruptured, sent him into the infirmary.
The patient insisted on walking downstairs to the cab, and on arrival at the infirmary stepped out of the cab into the ambulance chair.

Condition on Admissson.

The patient was a strong, well-made, burly man. The pulse was 132 , and the temperature $99^{\circ}$. He looked pallid, but was not at all collapsed. He complained of pain in the epigastrium and left hypochondrium, and was tender over that area. 'The abdomen moved fairly well on respiration, but there was some in the left flank.

A turpentine enema was ordered, and hot fomentation applied to the abdomen. At 4 p.m., when I saw him again, his 